\title{
Validation and proposed modification of the 8th edition American Joint Committee on Cancer staging system for patients with esophageal neuroendocrine neoplasms: Evaluation of a revised lymph node classification
}

\author{
HAIHONG WANG $^{1 *}$, YAOBING CHEN ${ }^{2 *}$, GUOLIANG PI $^{3 *}$, YING ZHU ${ }^{1}$, \\ SHENGLI YANG ${ }^{1}$, HONG MEI $^{4}$, ZHENYU LIN $^{1}$ and TAO ZHANG ${ }^{1}$
}

\begin{abstract}
${ }^{1}$ Cancer Center, Union Hospital, Tongji Medical College, Huazhong University of Science and Technology, Wuhan, Hubei 430022; ${ }^{2}$ Institute of Pathology, Tongji Hospital, Tongji Medical College, Huazhong University of Science and Technology, Wuhan, Hubei 430030; ${ }^{3}$ Department of Radiation Oncology, Hubei Cancer Hospital, Tongji Medical College, Huazhong University of Science and Technology, Wuhan, Hubei 430079; ${ }^{4}$ Pediatric Surgery, Union Hospital, Tongji Medical College, Huazhong University of Science and Technology, Wuhan, Hubei 430022, P.R. China
\end{abstract}

Received July 3, 2019; Accepted January 23, 2020

DOI: 10.3892/ol.2020.11480

\begin{abstract}
There is currently no universally accepted staging system for esophageal neuroendocrine neoplasms (ENENs). In the present study, patients with ENENs, identified from the Surveillance, Epidemiology, and End Results registry (SEER) $(\mathrm{n}=191$ patients) and the multicentric series ( $\mathrm{n}=51$ patients), were stratified to assess the validity of the 8th American Joint Committee on Cancer (AJCC) staging systems, particularly for esophageal squamous cell carcinoma and esophageal adenocarcinoma. The Kaplan-Meier method was used to assess disease-specific survival (DSS), according to the Tumor-Node-Metastasis (TNM) status, and the Cox model was
\end{abstract}

Correspondence to: Dr Zhenyu Lin or Dr Tao Zhang, Cancer Center, Union Hospital, Tongji Medical College, Huazhong University of Science and Technology, 1277 Jiefang Avenue, Wuhan, Hubei 430022, P.R. China

E-mail:whxhlzy@hust.edu.cn

E-mail: taozhangxh@hust.edu.cn

*Contributed equally

Abbreviations: ENENs, esophageal neuroendocrine neoplasms; SEER, Surveillance, Epidemiology, and End Results registry; AJCC, American Joint Committee on Cancer; DSS, disease-specific survival; TNM, Tumor-Node-Metastasis; pTNM, pathological Tumor-Node-Metastasis stage groups; Nr, revised $\mathrm{N}$; EAC, esophageal adenocarcinoma; ESCC, esophageal squamous cell carcinoma; ypTNM, postneoadjuvant pathologic stage groups; cTNM, clinical stage groups; HR, hazard ratio; CI, confidence interval; C-index, concordance index

Key words: neoplasm staging, AJCC, validation and modification, ENENs, SEER applied to evaluate differences in prognosis after adjustment for potential confounders. For the 8th AJCC staging classifications, only the pathological stage groups (pTNM) conferred increased hazard ratios from stage I to stage IV, with overlaps between adjacent stages. According to the current findings, the regional lymph nodes involvement status other than the current $\mathrm{N}$ classification was a significant predictor of DSS. Consequently, a revised $\mathrm{N}(\mathrm{Nr})$ classification was proposed and therefore a new TNrM staging system was adopted, for which progressively poorer DSS associated with increasing stage was observed. Moreover, the concordance index with the modified staging system was slightly higher in patients with ENENs from the SEER registry compared with that of the 8th pTNM system. In conclusion, lymph node status, rather than the number of positive lymph nodes, was a marker of poorer DSS and the modified staging system provided an easier and more accurate staging tool. The present results indicate that revisions to the current staging classifications may be improve the assessment of patient prognosis.

\section{Introduction}

Esophageal neuroendocrine neoplasms (ENENs) are exceedingly uncommon but increasingly prevalent malignancies with high heterogeneity. In 1997 , only three patients $(0.05 \%)$ were documented in the esophagus among 6,122 cases of gastrointestinal carcinoid tumors (1); In Korea, ENENs only accounted for 1.3-1.4\% of the gastrointestinal NENs reported from 2012 to $2014(2,3)$. They are characterized by distinct histological features and tumor behavior compared with esophageal adenocarcinoma (EAC) and esophageal squamous cell carcinoma (ESCC) $(4,5)$. Owing to its rarity, only a few cases concerning the ENENs have been reported in the literature in the previous two decades, with most focusing on the clinicopathological characteristics $(3,5-7)$ and treatment $(8,9)$. As a result, a staging system that can accurately provide 
prognostic information and stratify patients by risk are far from being well established up to date. The Tumor-Node-Metastasis (TNM) classification is the most frequently used indicator of outcomes for malignancies $(10,11)$. Several studies have indicated that the 7th American Joint Committee on Cancer (AJCC) TNM staging system for EACC may also be applied to describe ENENs $(5,6)$. However, this classification for ENENs never achieved widespread acceptance, due to the relatively small population size. More recently, the 8th AJCC staging system on esophageal cancer, which was introduced in 2016, has been recommended to replace the old version. The current (8th) TNM classification presents three classifications separately for both EAC and ESCC: The classic pathological stage groups (pTNM) expanded from the seventh edition foundation, the newly introduced postneoadjuvant pathologic stage groups (ypTNM), and clinical stage groups (cTNM) (Tables SI and SII) (12-14). The definition of T was optimized in the 8th classification, and the descriptors of $\mathrm{N}$ and $\mathrm{M}$ remained unchanged. The current $\mathrm{N}$ classification has already incorporated the number of lymph nodes with metastasis into the staging protocols. The $\mathrm{N}$ classification for solid cancer of various origins, including gastroenteropancreatic neuroendocrine neoplasms, only distinguishes between node-negative and node-positive disease, and the lymph nodes status was demonstrated to exhibit good discrimination of prognosis (15-17). However, to the best of our knowledge, the prognostic value of both the 8th AJCC staging system and lymph nodes status has not been validated in ENENs, particularly within large population-based samples.

In the present study, population-based data was used to validate the current TNM staging systems, and a new modified staging classification which adopted the revised $\mathrm{N}$ definition was tested that would address the weaknesses associated with the staging system and predict the prognosis of patients with ENENs more accurately and quickly.

\section{Patients and methods}

Patients and data collection. The data used in the present study was retrieved between 1973 and 2015 (months unavailable) from the Surveillance, Epidemiology, and End Results registry (SEER) database (seer.cancer.gov) of the US National Cancer Institute. Eligible patients were those who were diagnosed with pathologically confirmed ENENs (Appendix S1). The demographics and tumor variables (race, sex, age, location, grade, survival months and SEER cause-specific death classification) were retrieved from SEER. A tumor location (upper, middle and lower) was assigned for each patient on the basis of retrospective review of SEER data. The data on primary tumor size, depth of invasion, local extension, lymph node involvement and presence/absence of distant metastases were used to determine the TNM categories (Appendix S1). Individuals with unknown follow-up information, unknown cancer stage at diagnosis and who had presented with other primary tumors were excluded. The patients with unknown extent of tumor or lymph node status were included in the study if they had distant metastases. A total of 300 eligible patients with pathologically confirmed ENENs were identified from the SEER database. A total of 63 patients who had other primary cancer types, 45 patients with unknown cancer stage at diagnosis and one patient with unknown follow-up information, were excluded; leaving a final cohort of 191 patients in the study (Table I).

Three Chinese centers provided data on the ENENs for the validation analysis: Wuhan Union Hospital (Wuhan; between January 2011 and June 2018; n=16), Hubei Cancer Hospital (Wuhan; from January 2009 to December 2016; $n=6$ ), and Wuhan Tongji Hospital (Wuhan; from Jan 1, 2012, to Jun 1, 2017, $N=29$ ). The inclusion and exclusion criteria were identical to those used in the development cohort. A total of 51 patients from the multicentric database were included in the validation analysis. In addition to the demographics and tumor variables aforementioned, the Ki67 and cell morphology were also investigated Reports with no mention of small-cell morphology present or large-cell morphology were grouped into the non-small-cell classification. The classification of cancer location was based on the distance from the epicenter of the tumor to the incisors as follows: Upper ( $>15$ and $\leq 24 \mathrm{~cm})$; middle ( $>24$ and $\leq 33 \mathrm{~cm}$ ); and lower ( $>33$ and $\leq 42 \mathrm{~cm})$. Approval for the study protocol was obtained by the Institutional Ethics Committee of the Tongji Medical College, Huazhong University of Science and Technology. Written informed consent was provided all the patients for their data to be used for research.

Statistical analysis. Descriptive statistics were used to report the demographic characteristics of enrolled patients and stage distribution. The limited disease was defined as a neoplasm without distant metastases, and the extensive disease was defined as a neoplasm that transfer to parts of the body remote from the primary tumor. Since patients with distant metastasis were categorized in the advanced stage, only the $\mathrm{T}$ and $\mathrm{N}$ statuses of patients with limited disease were reported. Patients who succumbed to causes other than cancer were censored at their date of death. Disease-specific survival (DSS) was estimated via the Kaplan-Meier method and the log-rank test was used to compare the survival curves between groups using SPSS software (version 22.0; IBM Corp.). The T, $\mathrm{N}$ and $\mathrm{M}$ categories were combined according to the current stage groups for EAC and ESCC. The potential overlaps in outcomes among groups were further investigated by modification of the current staging classifications. Multivariate analysis was performed to determine which $\mathrm{N}$ classifications were able to independently predict outcomes using the Cox proportional hazards regression. On the basis of the current findings, a $\mathrm{Nr}$ classification ( $\mathrm{Nr} 0$, negative lymph nodes; $\mathrm{Nr} 1$, positive lymph nodes) was proposed and the survival for all possible $\mathrm{T}$ and $\mathrm{Nr}$ combinations (such as T1Nr0M0, T1Nr1M0 and $\mathrm{T} 2 \mathrm{Nr} 0 \mathrm{M} 0$ ), was estimated; thus, a modified staging system for ENENs was devised. The Cox proportional hazards model was used to evaluate the association between each staging classification and DSS after adjusting for race, sex, age, tumor grade and tumor location. Only race, sex and age were adjusted in the Cox regression model for pTNM, given that the grade and tumor location had been assigned to the 8th AJCC pathological staging classifications. Hazard ratios (HRs) and 95\% confidence intervals (CIs) were calculated. Concordance indices were calculated to evaluate the discriminatory powers of the staging systems. A concordance index (C-index) of 1 represents perfect discrimination, and a $\mathrm{C}$-index of 0.5 denoted 
Table I. Baseline clinicopathological characteristics.

\begin{tabular}{|c|c|c|}
\hline Characteristic & SEER, $n=191$ & Multicentric database, $n=51$ \\
\hline Median age, years (IQR) & $65.0(57.5-72.0)$ & $60.0(53.5-67.5)$ \\
\hline \multicolumn{3}{|l|}{$\operatorname{Sex}, \mathrm{n}(\%)$} \\
\hline Male & $159(83.2)$ & $34(66.7)$ \\
\hline Female & $32(16.8)$ & $17(33.3)$ \\
\hline \multicolumn{3}{|l|}{ Race, n (\%) } \\
\hline White & $165(86.4)$ & $0(0.0)$ \\
\hline Black & $13(6.8)$ & $0(0.0)$ \\
\hline Other & $13(6.8)$ & $51(100.0)$ \\
\hline \multicolumn{3}{|l|}{ Location, n (\%) } \\
\hline Upper & $11(5.8)$ & $9(17.6)$ \\
\hline Middle & $30(15.7)$ & $21(41.2)$ \\
\hline Lower & $108(56.5)$ & $16(31.4)$ \\
\hline Unknown & $42(22.0)$ & $5(9.8)$ \\
\hline \multicolumn{3}{|l|}{ Grade, n (\%) } \\
\hline $\mathrm{G} 1-\mathrm{G} 2$ & $5(2.6)$ & $0(0.0)$ \\
\hline G3-G4 & $151(79.1)$ & $36(70.6)$ \\
\hline Unknown & $35(18.3)$ & $15(29.4)$ \\
\hline Median tumor size, cm (IQR) & $6.2(4.0-8.0)$ & $3.9(3.0-5.0)$ \\
\hline Median Ki67, (IQR) & NR & $0.80(0.70-0.90)$ \\
\hline Cell morphology, n (\%) & NR & \\
\hline Small cell & & $17(33.3)$ \\
\hline Non-small cell & & $34(66.7)$ \\
\hline \multicolumn{3}{|l|}{ T status ${ }^{\mathrm{a}}, \mathrm{n}(\%)$} \\
\hline $\mathrm{T} 1$ & $31 / 66(47.0)$ & $10 / 33(30.3)$ \\
\hline $\mathrm{T} 2$ & 7/66 (10.6) & $14 / 33(42.4)$ \\
\hline $\mathrm{T} 3$ & $19 / 66(28.8)$ & 9/33 (27.3) \\
\hline $\mathrm{T} 4 \mathrm{a}$ & $2 / 66(3.0)$ & $0 / 33(0.0)$ \\
\hline $\mathrm{T} 4 \mathrm{~b}$ & 7 /66 (10.6) & $0 / 33(0.0)$ \\
\hline \multicolumn{3}{|l|}{$\mathrm{N}$ status ${ }^{\mathrm{a}}, \mathrm{n}(\%)$} \\
\hline N0 & $38 / 66(57.6)$ & $13 / 33(39.4)$ \\
\hline N1 & $21 / 66(31.8)$ & $11 / 33(33.30)$ \\
\hline $\mathrm{N} 2$ & $6 / 66(9.1)$ & $8 / 33(24.2)$ \\
\hline N3 & $1 / 66(1.5)$ & $1 / 33(3.0)$ \\
\hline \multicolumn{3}{|c|}{ Regional lymph nodes involvement status ${ }^{\mathrm{a}}, \mathrm{n}(\%)$} \\
\hline Negative & $38 / 66(57.6)$ & $13 / 33(39.4)$ \\
\hline Positive & $28 / 66(42.4)$ & $20 / 33(60.6)$ \\
\hline \multicolumn{3}{|l|}{ M status, n $(\%)$} \\
\hline M0 & $66(34.6)$ & $33(64.7)$ \\
\hline M1 & $125(65.4)$ & $18(35.5)$ \\
\hline \multicolumn{3}{|l|}{ pTNM for ESCC, n (\%) } \\
\hline I & $23(12.0)$ & $5(9.8)$ \\
\hline IA & $8(4.2)$ & $0(0.0)$ \\
\hline IB & $15(7.8)$ & $5(9.8)$ \\
\hline II & $19(9.9)$ & $10(19.6)$ \\
\hline IIA & 7 (3.7) & $6(11.8)$ \\
\hline IIB & $12(6.2)$ & $4(7.8)$ \\
\hline III & $16(8.4)$ & $17(33.3)$ \\
\hline IIIA & $4(2.1)$ & $8(15.7)$ \\
\hline IIIB & $12(6.3)$ & $9(17.6)$ \\
\hline IV & $133(69.6)$ & $19(37.3)$ \\
\hline IVA & $8(4.2)$ & $1(2.0)$ \\
\hline IVB & $125(65.4)$ & $18(35.3)$ \\
\hline
\end{tabular}


Table I. Continued.

\begin{tabular}{|c|c|c|}
\hline Characteristic & SEER, $n=191$ & Multicentric database, $n=51$ \\
\hline \multicolumn{3}{|l|}{ pTNM for EAC, n (\%) } \\
\hline I & $23(12.0)$ & $5(9.8)$ \\
\hline IA & $8(4.2)$ & $0(0.0)$ \\
\hline IB & $1(0.5)$ & $1(2.0)$ \\
\hline $\mathrm{IC}$ & $14(7.3)$ & $4(7.8)$ \\
\hline II & $19(9.9)$ & $10(19.6)$ \\
\hline IIA & $2(1.0)$ & $6(11.8)$ \\
\hline IIB & $17(8.9)$ & $4(7.8)$ \\
\hline III & $16(8.4)$ & $17(33.3)$ \\
\hline IIIA & $4(2.1)$ & $8(15.7)$ \\
\hline IIIB & $12(6.3)$ & $9(17.6)$ \\
\hline IV & $133(69.6)$ & $19(37.3)$ \\
\hline IVA & $8(4.2)$ & $1(2.0)$ \\
\hline IVB & $125(65.4)$ & $18(35.3)$ \\
\hline \multicolumn{3}{|l|}{ cTNM for ESCC, n (\%) } \\
\hline $\mathrm{I}$ & $31(16.2)$ & $7(13.7)$ \\
\hline II & $15(7.9)$ & $13(25.5)$ \\
\hline III & $10(5.2)$ & $12(23.5)$ \\
\hline IV & 135 (70.7) & $19(37.3)$ \\
\hline IVA & $10(5.2)$ & $1(2.0)$ \\
\hline IVB & $125(65.4)$ & $18(35.3)$ \\
\hline \multicolumn{3}{|l|}{ cTNM for EAC, n (\%) } \\
\hline I & $23(12.0)$ & $5(9.8)$ \\
\hline II & $10(5.2)$ & $8(15.7)$ \\
\hline IIA & $8(4.2)$ & $2(3.9)$ \\
\hline IIB & $2(1.0)$ & $6(11.8)$ \\
\hline III & $19(9.9)$ & $11(21.6)$ \\
\hline IV & $139(72.8)$ & $27(52.9)$ \\
\hline IVA & $14(7.3)$ & $9(17.6)$ \\
\hline IVB & $125(65.4)$ & $18(35.3)$ \\
\hline \multicolumn{3}{|l|}{ Modified staging system, n (\%) } \\
\hline I & $23(12.0)$ & $5(9.8)$ \\
\hline II & $11(5.8)$ & $8(15.7)$ \\
\hline III & $25(13.1)$ & $20(39.2)$ \\
\hline IV & $132(69.1)$ & $18(35.3)$ \\
\hline IVA & $7(3.7)$ & 0 \\
\hline IVB & $125(65.4)$ & $18(35.3)$ \\
\hline Median follow-up time, months (IQR) & $6.0(2.0-16.0)$ & $7.0(4.0-16.0)$ \\
\hline Median DSS time, months (95\% CI) & $9.0(6.6-11.4)$ & $16.0(11.5-20.5)$ \\
\hline Median DSS time for limited disease, months (95\% CI) & $22.0(15.2-28.8)$ & $19.0(13.9-24.1)$ \\
\hline Median DSS time for extensive disease, months $(95 \% \mathrm{CI})$ & $6.0(4.3-7.7)$ & $6.0(4.4-7.6)$ \\
\hline
\end{tabular}

${ }^{\mathrm{a}} \mathrm{T}$ and $\mathrm{N}$ status for patients with metastatic disease not reported. IQR, interquartile range; G1, well differentiated; G2, moderately differentiated; G3, poorly differentiated; G4, undifferentiated; T, tumor; N, lymph node; M, metastasis; ESCC, esophageal squamous cell carcinoma; EAC, esophageal adenocarcinoma; NR, not reported; pTNM, pathologic stage groups; cTNM, clinical stage groups; DSS, disease-specific survival; CI, confidence interval; SEER, Surveillance, Epidemiology, and End Results.

agreement by chance alone. Analyses were performed using SPSS (version 22.0; IBM Corp.), RStudio [version 1.1.456; (18)] and $\mathrm{R}$ [version 3.5.1; (19)] software. $\mathrm{P}<0.05$ was considered to indicate a statistically significant difference.

\section{Results}

Patient and tumor characteristics. A final cohort of 191 patients from the SEER database were included in the 

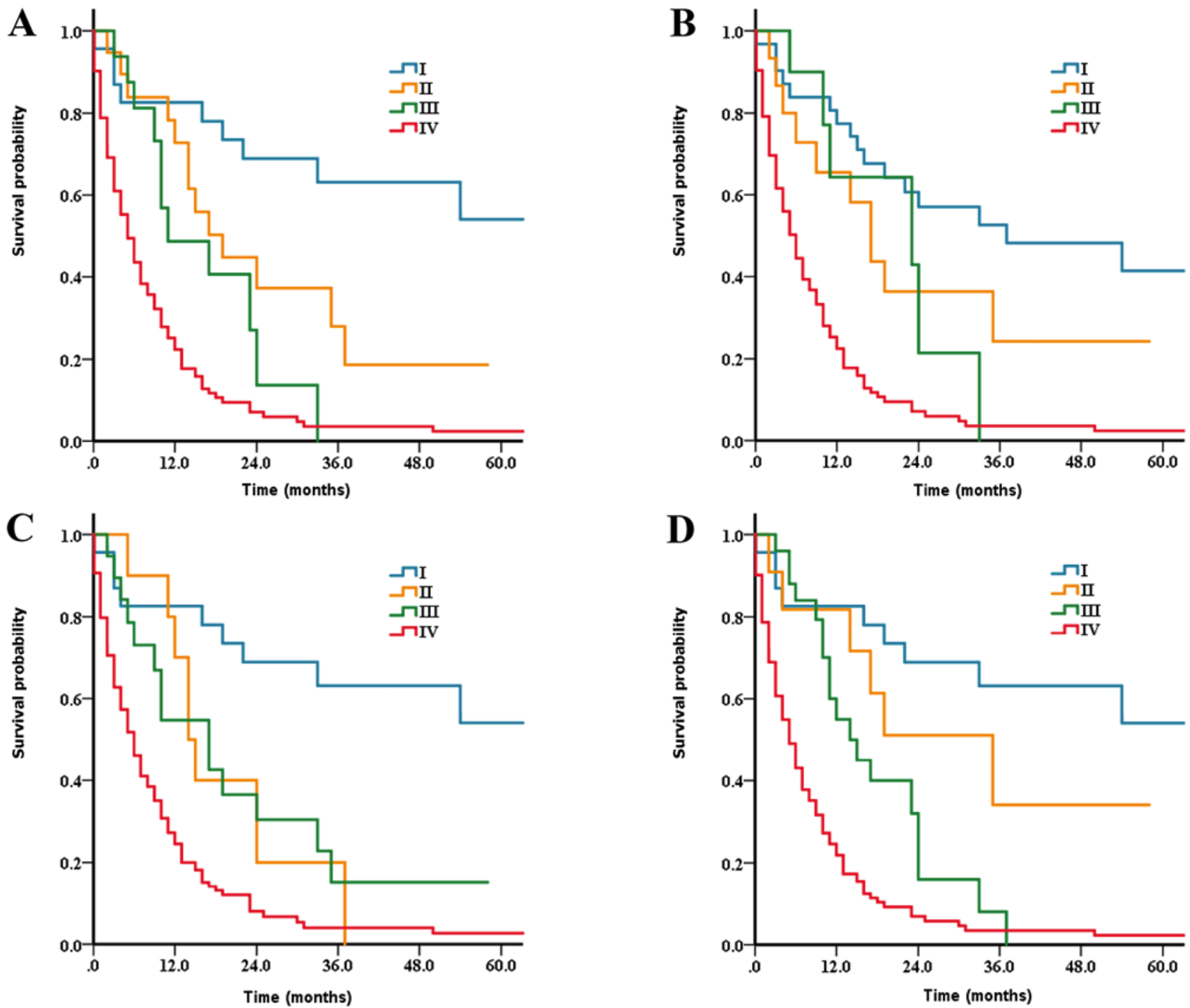

Figure 1. DSS curves of different staging classifications for patients with esophageal neuroendocrine neoplasms from the Surveillance, Epidemiology, and End Results database. DSS curves for the 8th AJCC (A) pathological staging system and (B) clinical staging system for esophageal squamous cell carcinoma. DSS curves for (C) the 8th AJCC clinical staging system for esophageal adenocarcinoma and (D) the modified staging system. DSS, disease-specific survival; AJCC, American Joint Committee on Cancer.

study (Table I). The median age at diagnosis was 65.0 years (range, 57.5-72.0 years) with a male to female ratio of 5.0. The majority of tumors were located in the middle (15.7\%) or lower $(56.5 \%)$ esophagus, and $79.1 \%$ of the tumors were poorly differentiated. A total of $125(65.4 \%)$ patients had metastatic disease. Of the entire study population, the median DSS was 9.0 months. The median DSS for limited disease and extensive disease were 22.0 and 6.0 months, respectively.

The median age for the multicentric database was 60.0 years (range, 53.5-67.5 years) with a male to female ratio of 2.0 . Notably, $\sim 73 \%$ of patients had a tumor located in the middle (41.2\%) or lower (31.4\%) of esophagus and $70.6 \%$ of the tumors were poorly differentiated. Small cell carcinoma accounted for $33.3 \%$ of all types of ENENs in the present study. A total of 18 (35.3\%) patients had metastatic disease. The median DSS times for the whole cohort, limited disease and extensive disease were 16.0, 19.0 and 6.0 months, respectively (Table I).
Pathologic stage groups and survival. Survival curves according to current pTNM were analyzed. As presented in Table I, the proportion of stage groups without further subgroup classifications for EAC and ESCC was the same. The distribution was as follows in the SEER database; Stage I (12.0\%); stage II (9.9\%); stage III (8.4\%) and stage IV (69.6\%). Multicenter series was as follows; Stage I (9.8\%); stage II (19.6\%); stage III (33.3\%) and stage IV (37.3\%). The Kaplan-Meier analysis revealed progressively poorer DSS with more advanced stages in both EAC and ESCC [median DSS: Stage I (80.1 months); stage II (25.6 months); stage III (16.3 months) and stage IV (9.6 months); Fig. 1A], with overlaps between stage II and stage III disease for the SEER series ( $\mathrm{P}=0.177$ for stage II vs. stage III). For the multicentric series (Fig. 2A), there were also overlaps in the survival of patients with stages II and III disease $(\mathrm{P}=0.402)$. A significant increase in HRs was observed in stages I-III in the SEER series compared with stage IV, via multivariate analysis (Table II). Furthermore, multivariate analysis in the multicentric series 

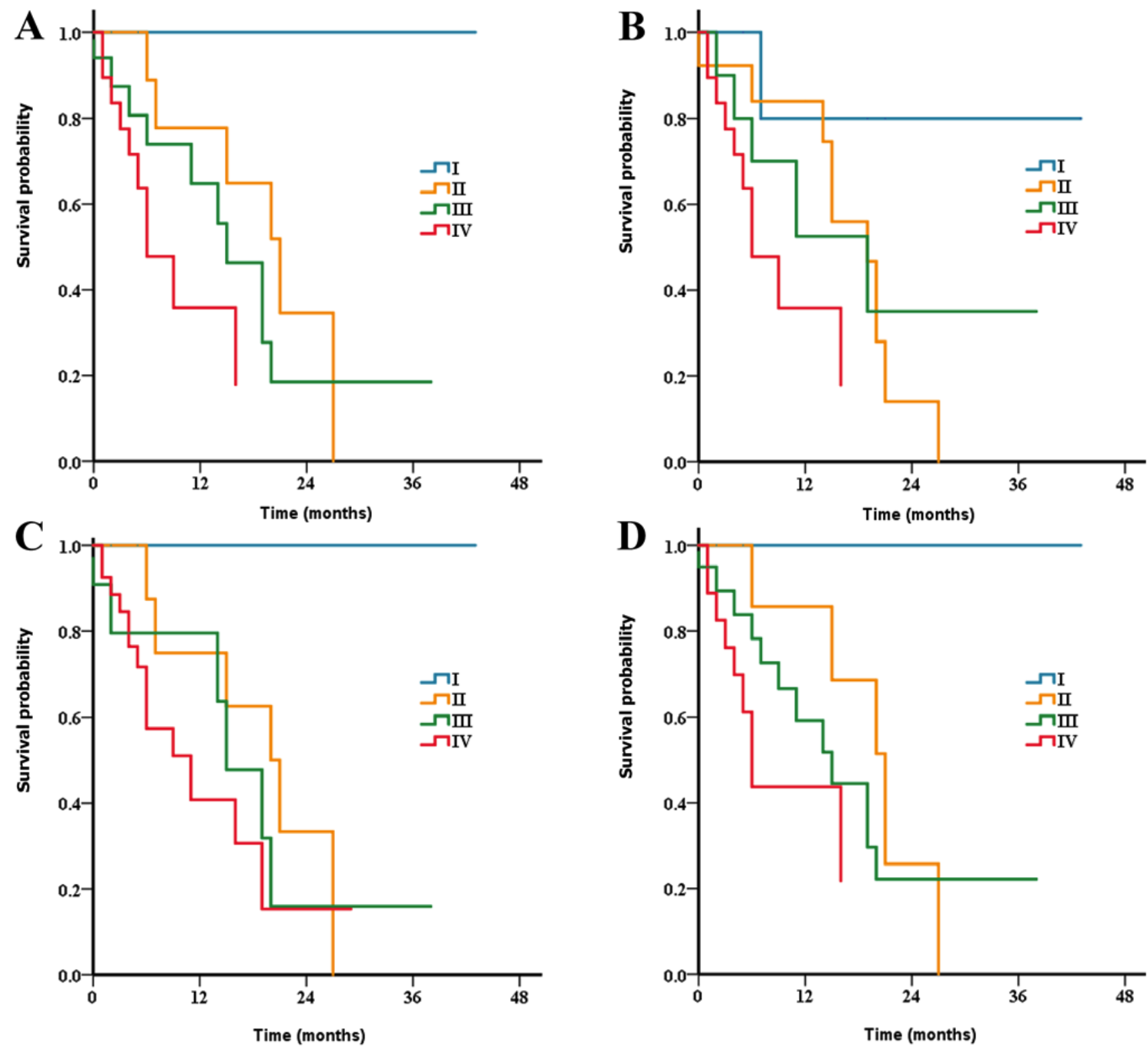

Figure 2. DSS curves of different staging classifications for patients with esophageal neuroendocrine neoplasms from the multicentric database. DSS curves for the 8th AJCC (A) pathological staging system and (B) clinical staging system for esophageal squamous cell carcinoma. DSS curves for (C) the 8th AJCC clinical staging system for esophageal adenocarcinoma and (D) the modified staging system. DSS, disease-specific survival; AJCC, American Joint Committee on Cancer.

also demonstrated increased risk of mortality of the patients in stages I-IV (Table III).

Clinical stage groups and survival. According to the current cTNM for ESCC, it is notable that overlaps in DSS were observed between stage I and II diseases, and between stage II and III diseases for both the SEER series (Fig. 1B) and the multicentric database (Fig. 2B). Patients with stage II disease and stage III disease exhibited similar survival (median DSS time from SEER series: 17 vs. 23 months, $\mathrm{P}=0.992$; median DSS from multicentric series: 19 vs. 19 months, $\mathrm{P}=0.697$ ). Compared with stage IV disease, the HR of stage II was comparable to that of stage III in both SEER series (Stages II and III HRs, 0.31 and 0.31, respectively; Table II) and multicentric series (stages II and III HRs, 0.38 and 0.49 , respectively; Table III) by multivariate analysis.
According to the current cTNM for EAC, overlaps were also revealed between the stage II and III diseases for both the SEER series (Fig. 1C) and the multicentric database (Fig. 2C). Compared with stage IV disease, the HRs for stages III, II and I diseases were 0.40 (95\% CI, 0.22-0.71; $\mathrm{P}=0.002), 0.41$ (95\% CI, 0.19-0.86; $\mathrm{P}=0.019)$ and 0.13 (95\% CI, 0.06-0.27; $\mathrm{P}<0.001)$, respectively (Table II). Thus, the risk of death increased in stage II, decreased from stage IV to III and decreased further in stage I for the patients in the SEER series. There was no significant difference in HR between stages II and III for the multicentric series; compared with stage II: Stage III (HR, 1.46; 95\% CI, 0.36-5.87; $\mathrm{P}=0.598$ ) (Table III).

Grouping of ENENs by lymph node status. The Kaplan-Meier analysis exhibited no worsening DSS as the number of positive lymph nodes increased for both SEER series and multicentric 


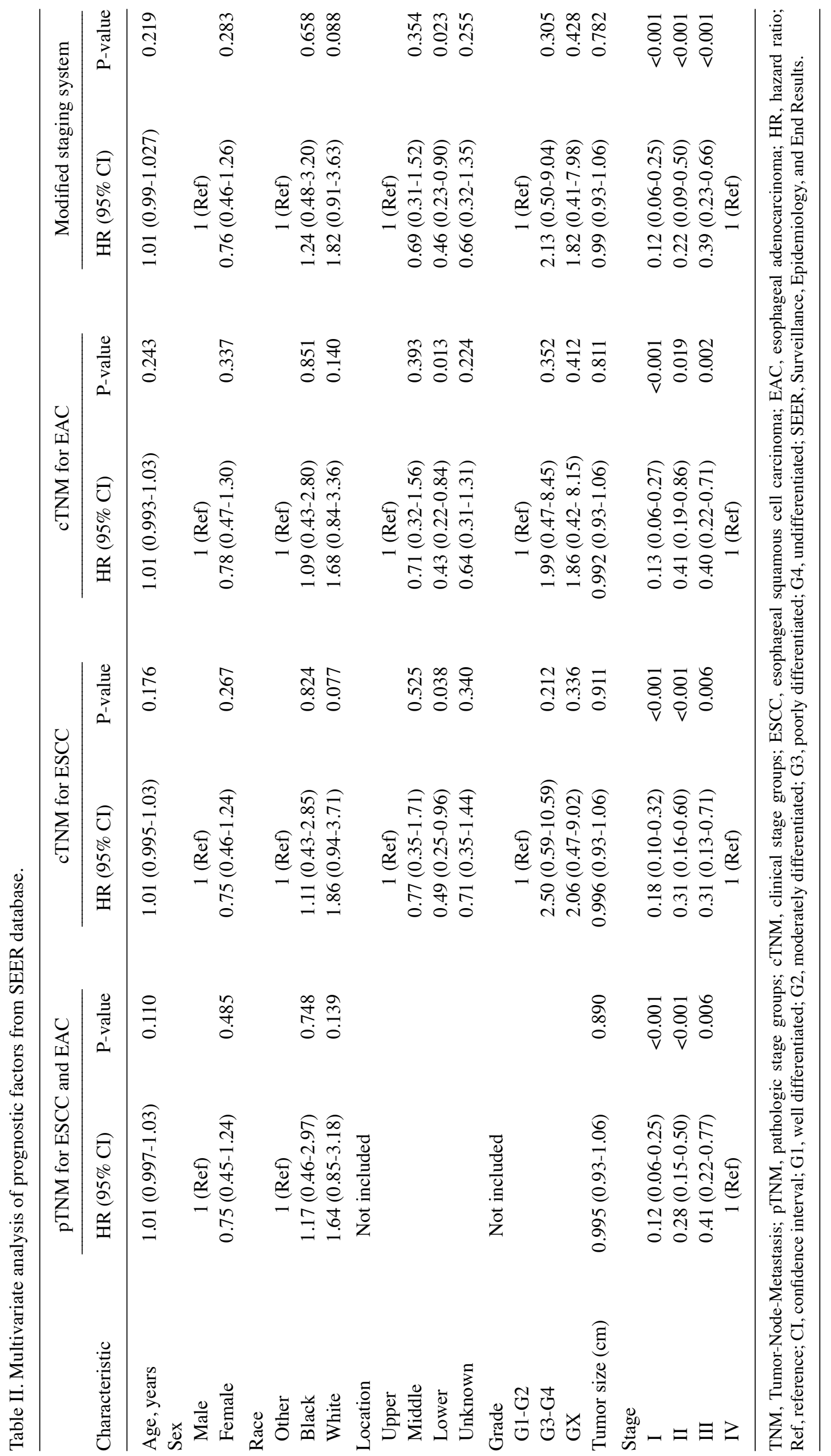




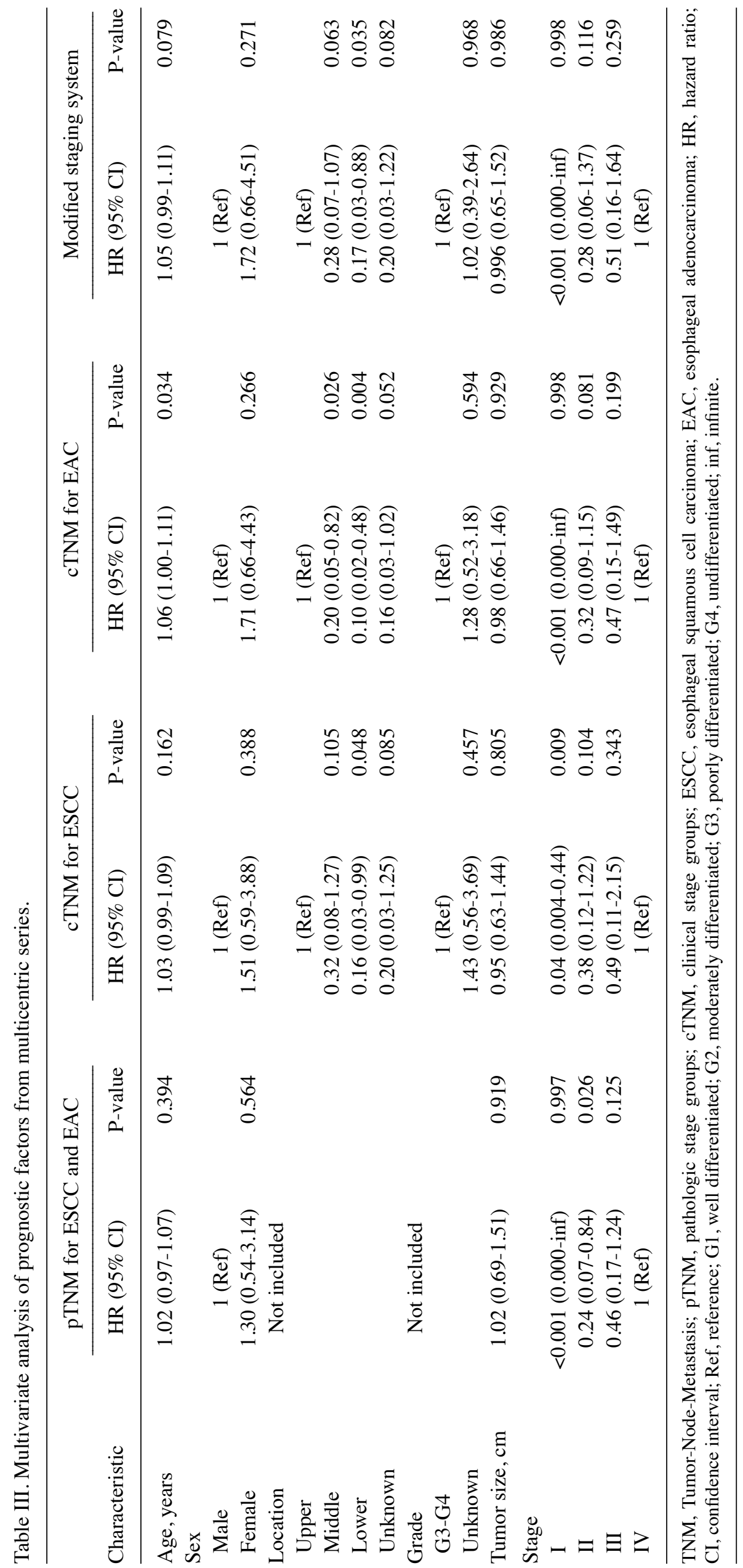


series (Fig. S1). Further multivariate analysis revealed no increased HRs of death from stage N0 to stage N3 for the patients without metastases from the SEER series; compared with stage N0 disease: N1 (HR, 2.70; 95\% CI, 1.33-5.49; $\mathrm{P}=0.006$ ); N2 (HR, 0.83; 95\% CI, 0.18-3.92; $\mathrm{P}=0.817$ ); and N3 (HR <0.001, $\mathrm{P}=0.997)$ (Table SIII). However, patients with $\mathrm{Nr} 0$ exhibited a significantly improved DSS time compared with patients with $\mathrm{Nr} 1$ disease according to the univariate and multivariate analysis for both two cohorts (Table SIV and SV).

Proposal and validation of new TNrM stage groups. The Kaplan-Meier survival curves, including all $\mathrm{T}, \mathrm{Nr}$ and $\mathrm{M}$ combinations, indicated multiple overlaps across the groups for patients with ENENs from the SEER series (Fig. S2). These curves demonstrated that the best outcome was attained by patients with $\mathrm{T} 1 \mathrm{Nr} 0$ disease (1-year survival, $82.6 \%$; 2-year survival, 68.8\%), which was defined as stage I. The next best similar survival between patients with $\mathrm{T} 2 \mathrm{Nr} 0$ and $\mathrm{T} 3 \mathrm{Nr} 0$ disease (1-year survival, 100.0 and $77.8 \%$, respectively; 2-year survival, 50.0 and $51.9 \%$, respectively; $\mathrm{P}=0.946$ ) indicates that the two stages should be defined as stage II disease. There was an overlap of survival in patients with T1Nr1 (1-year survival, $62.5 \%$; 2-year survival, $18.8 \%$ ) and $\mathrm{T} 2 \mathrm{Nr} 1$ (1-year survival, $40.0 \%$; 2-year survival, $20.0 \%)$ disease $(\mathrm{P}=0.417)$, as well as in patients with $\mathrm{T} 2 \mathrm{Nr} 1$ and $\mathrm{T} 3 \mathrm{Nr} 1$ disease (1-year survival, $61.7 \%$; 2-year survival, 20.6\%) $(\mathrm{P}=0.108)$. In addition, patients with T4aNrl disease had similar survival compared with stage T3Nr1 disease $(\mathrm{P}=0.205)$. The results suggest that the new staging system should be modified to adopt the definition of stage III disease (T1-3Nr1M0 and T4aNr0-1M0). Patients with T4b disease (1-year survival, $0 \%$; 2-year survival, $0 \%$ ) or M1 disease (1-year survival, 23.3\%; 2-year survival, 7.3\%) exhibited the poorest survival, which should therefore be defined as stage IV. Thus, new modified staging definitions were adopted (Table SVI), which were mostly similar to the staging classifications for the gastrointestinal neuroendocrine neoplasms. Overall, DSS was significantly different across stages for both SEER series $(\mathrm{P}<0.001)$ and multicentric database $(\mathrm{P}=0.034)$ (Figs. 1D and 2D) with persistent overlaps in combined stage. There was an expected decrease in survival times as tumor stage increased with HRs of 0.12 (95\% CI, 0.06-0.25), 0.22 (95\% CI, 0.09-0.50), and 0.39 (95\% CI, 0.23-0.66) for stages I, II, and III, respectively, compared with stage IV in the patients from SEER database (Table II). Decreased DSS time was also observed for stages I-III compared with stage IV, for the cohort from multicentric database, with HRs of $<0.001$ (95\% CI, 0.000-inf), 0.28 (95\% CI, 0.06-1.37), and 0.51 (95\% CI, 0.16-1.64), respectively, (Table III).

The C-indices of different staging systems are presented in Table SVII. The respective C- indices using the 8th pTNM and the modified staging systems for patients with ENENs were as follows: SEER series; 0.656 (95\% CI, 0.607-0.705) and 0.659 (95\% CI, 0.610-0.708), respectively; multicentric database, 0.69 (95\% CI, 0.561-0.819) and 0.688 (95\% CI, 0.561-0.815), respectively.

\section{Discussion}

Currently, there is no specific staging system for the ENENs, and the present study was the first consolidation of a data-based process for the revision of the 8th AJCC staging classification both for EAC and ESCC to assess DSS in this exceedingly uncommon and increasingly prevalent cancer type (1-3). The proposed system may help to better discriminate prognosis of patients with ENENs and guide the evaluation of current and novel treatments for this disease.

Due to the unavailability of the information on postneoadjuvant therapy from the SEER database, the 8th AJCC ypTNM were not used to assess the prognostic value. The analysis revealed that the 8th AJCC pTNM system has an improved potential for the discrimination of prognosis compared with the current AJCC cTNM systems. It is unlikely that stages II and III represent distinct prognostic categories in view of the fact that survival outcomes are not significant for both groups according to the current cTNM systems. However, further subgroup analysis suggested the consistency of the outcomes among the substages became unclear based on the pTNM systems (Figs. S3 and S4). Consequently, the present study revealed that the existing TNM staging classifications may not optimally distinguish survival outcomes among patients with ENENs. Moreover, the clinical stage grouping prior to treatment decision has received increasing attention since the wide application of modern imaging and endoscopic technologies, thus warranting further modifications and validation of an easier and more accurate cTNM staging groups for ENENs. In addition, multivariate analysis indicated that the lymph node status (excluding the number of lymph nodes with metastasis) was a significant prognostic factor, supporting the adoption of the $\mathrm{Nr}$ definition in the modified staging system. Therefore, a modified cTNM to the 8th AJCC staging classifications was proposed, which maintains the $\mathrm{T}$ and $\mathrm{M}$ definitions but adopts a new $\mathrm{Nr}$ definition, similar to the gastrointestinal staging classifications (stage I, T1N0M0; stage II, T2-T3N0M0; stage III, T1-T3N1MO and T4NanyM0; and stage IV, TanyNanyM1). The modified staging system was confirmed using two relatively large ENEN series, and the risk of mortality uniformly progressed from class I to IV for both cohorts. Moreover, the discrimination ability for tumor-associated mortality (measured by the $\mathrm{C}$-index) was slightly better or comparable for the modified staging system, relative to the pTNM system. Due to the increased application of computed tomographic scans and endoscopic technologies, it was relatively easy to assess the regional lymph node involvement status. However, given that some patients did not have lymph node dissection or presented with insufficient lymph node dissection, the number of positive lymph nodes was difficult to assess. Consequently, the current findings suggest that the modified staging classification may be more suitable and easier for ENENs and can be adopted in clinical practice in the future.

Currently, there is no consensus on the guidelines for the management of ENENs due to their rarity and heterogeneity. The establishment of an accurate and easy staging is the first step in optimizing treatment and outcomes for patients with ENENs. Accumulating evidence has supported the application of surgical resection as an effective treatment option for patients with ENEN with limited disease. Notably, cT4b tumors with invasion of the heart, great vessels trachea, or adjacent organs including liver, pancreas, lung and spleen are considered to be unresectable (20). Therefore, the modified staging groups may help to distinguish the patients with unresectable disease from 
the patients without distant metastasis. Moreover, the present population-based study enabled us to detect differences in outcomes, particularly among patients with limited disease without lymph nodes metastasis, who have a relatively good prognosis and may not experience rapid disease progression, which provides a theoretical basis for future stratified treatment. Moreover, the median DSS time for limited disease in the present study ranged from 19-22 months, and for extensive disease, the median DSS was $\sim 6$ months, which supports a study on small cell carcinoma of esophagus reported by Luo et al (15).

The present study has several limitations. The rarity of ENETs makes it difficult to accumulate a large cohort for evaluation, which may explain the existence of overlaps among stages using the modified staging system results in a decrease of statistical power to detect significant differences among substages. The present study was also limited by its retrospective nature. Therefore, additional prospective studies with a larger sample size will be required to validate the modified staging system.

In conclusion, although the 8th AJCC pTNM classification allows for the discrimination of clinical outcomes in patients with ENENs, the present findings suggested that this system should be further refined. A modified clinical staging system was proposed by maintaining the $\mathrm{T}$ and $\mathrm{M}$ definitions of the current AJCC staging system and adopting the $\mathrm{Nr}$ classification, which demonstrated a significant association with DSS. Although the modified staging system may be more accurate and easier in predicting the prognosis of ENENs, the current findings still need to be replicated in other cohorts before adoption in clinical practice.

\section{Acknowledgements}

Not applicable.

\section{Funding}

The present study was funded by The National Natural Science Foundation of China (grant no. 81874061), Chinese Society of Clinical Oncology-Shiyao Cancer Research Fund (grant no. Y-sy2019-009), Hubei Provincial Natural Science Foundation Guiding Project (grant no. 2018CFC846) and The 7th Wuhan Young and Middle-aged Backbone Talent of Medical Training Project 2019 (grant nos. 2019 and 87).

\section{Availability of data and materials}

The datasets used and/or analyzed during the present study are available from the corresponding author upon reasonable request.

\section{Authors' contributions}

All authors conceived and designed the present study and drafted the initial manuscript. YC, GP, SY, ZL and TZ provided the study material and patient information. HW, YC, GP, SY and $Y Z$ acquired the data. Statistical analysis was performed by HW, YC, GP, HM, ZL and TZ. All authors have read and approved the final manuscript.

\section{Ethics approval and consent to participate}

Approval for the study protocol was obtained by the Institutional Ethics Committee of the Tongji Medical College, Huazhong University of Science and Technology (approval no. S1172). Written informed consent was obtained from all the patients for their data to be used for research.

\section{Patient consent for publication}

Not applicable.

\section{Competing interests}

The authors declare that they have no competing interests.

\section{References}

1. Modlin IM and Sandor A: An analysis of 8305 cases of carcinoid tumors. Cancer 79: 813-829, 1997.

2. Gastrointestinal Pathology Study Group of Korean Society of Pathologists; Cho MY, Kim JM, Sohn JH, Kim MJ, Kim KM, Kim WH, Kim H, Kook MC, Park DY, et al: Current trends of the incidence and pathological diagnosis of gastroenteropancreatic neuroendocrine tumors (GEP-NETs) in Korea 2000-2009: Multicenter study. Cancer Res Treat 44: $157-165,2012$

3. Lee CG, Lim YJ, Park SJ, Jang BI, Choi SR, Kim JK, Kim YT, Cho JY, Yang CH, Chun HJ, et al: The clinical features and treatment modality of esophageal neuroendocrine tumors: A multicenter study in Korea. BMC Cancer 14: 569, 2014.

4. Wang KL, Yang Q, Cleary KR, Swisher SG, Correa AM, Komaki R, Ajani JA, Rashid A, Hamilton SR and Wu TT: The significance of neuroendocrine differentiation in adenocarcinoma of the esophagus and esophagogastric junction after preoperative chemoradiation. Cancer 107: 1467-1474, 2006.

5. Huang Q, Wu H, Nie L, Shi J, Lebenthal A, Chen J, Sun Q, Yang J, Huang L and Ye Q: Primary high-grade neuroendocrine carcinoma of the esophagus: A clinicopathologic and immunohistochemical study of 42 resection cases. Am J Surg Pathol 37: 467-483, 2013.

6. Deng HY, Ni PZ, Wang YC, Wang WP and Chen LQ: Neuroendocrine carcinoma of the esophagus: Clinical characteristics and prognostic evaluation of 49 cases with surgical resection. J Thorac Dis 8: 1250-1256, 2016.

7. Maru DM,Khurana H,Rashid A, Correa AM,Anandasabapathy S, Krishnan S, Komaki R, Ajani JA, Swisher SG and Hofstetter WL: Retrospective study of clinicopathologic features and prognosis of high-grade neuroendocrine carcinoma of the esophagus. Am J Surg Pathol 32: 1404-1411, 2008.

8. Deng HY, Li G, Luo J, Li XR, Alai G and Lin YD: The role of surgery in treating resectable limited disease of esophageal neuroendocrine carcinoma. World J Surg 42: 2428-2436, 2018.

9. Okuma HS, Iwasa S, Shoji H, Takashima A, Okita N, Honma Y, Kato K, Hamaguchi T, Yamada Y and Shimada Y: Irinotecan plus cisplatin in patients with extensive-disease poorly differentiated neuroendocrine carcinoma of the esophagus. Anticancer Res 34: 5037-5041, 2014.

10. Rice TW, Ishwaran H, Ferguson MK, Blackstone EH and Goldstraw P: Cancer of the esophagus and esophagogastric junction: An eighth edition staging primer. J Thorac Oncol 12: 36-42, 2017.

11. Rindi G, Falconi M, Klersy C, Albarello L, Boninsegna L, Buchler MW, Capella C, Caplin M, Couvelard A, Doglioni C, et al: TNM staging of neoplasms of the endocrine pancreas: Results from a large international cohort study. J Natl Cancer Inst 104: 764-777, 2012.

12. Rice TW, Ishwaran H, Hofstetter WL, Kelsen DP Apperson-Hansen C and Blackstone EH; Worldwide Esophageal Cancer Collaboration Investigators: Recommendations for pathologic staging (pTNM) of cancer of the esophagus and esophagogastric junction for the 8th edition AJCC/UICC staging manuals. Dis Esophagus 29: 897-905, 2016. 
13. Rice TW, Ishwaran H, Kelsen DP, Hofstetter WL, Apperson-Hansen C and Blackstone EH; Worldwide Esophageal Cancer Collaboration Investigators: Recommendations for neoadjuvant pathologic staging (ypTNM) of cancer of the esophagus and esophagogastric junction for the 8th edition AJCC/UICC staging manuals. Dis Esophagus 29: 906-912, 2016.

14. Rice TW, Ishwaran H, Blackstone EH, Hofstetter WL, Kelsen DP and Apperson-Hansen C; Worldwide Esophageal Cancer Collaboration Investigators: Recommendations for clinical staging (cTNM) of cancer of the esophagus and esophagogastric junction for the 8th edition AJCC/UICC staging manuals. Dis Esophagus 29: 913-919, 2016.

15. Luo G, Javed A, Strosberg JR, Jin K, Zhang Y, Liu C, Xu J, Soares K, Weiss MJ, Zheng L, et al: Modified staging classification for pancreatic neuroendocrine tumors on the basis of the American joint committee on cancer and European neuroendocrine tumor society systems. J Clin Oncol 35: 274-280, 2017.
16. Kim MK, Warner RR, Roayaie S, Harpaz N, Ward SC, Itzkowitz S and Wisnivesky JP: Revised staging classification improves outcome prediction for small intestinal neuroendocrine tumors. J Clin Oncol 31: 3776-3781, 2013.

17. Martin JA, Warner RR, Wisnivesky JP and Kim MK: Improving survival prognostication of gastroenteropancreatic neuroendocrine neoplasms: Revised staging criteria. Eur J Cancer 76: 197-204, 2017.

18. RStudio Team: RStudio: Integrated Development for R. RStudio, Inc., Boston, MA, 2015. http://www.rstudio.com/

19. R Core Team: R: a language and environment for statistical computing. R Foundation for Statistical Computing, Vienna, 2012. http://www.R-project.org/

20. Cuccurullo V and Mansi L: AJCC cancer staging handbook: From the AJCC cancer staging manual (7th edition). Eur J Nuclear Med Mol Imaging 38: 408-408, 2011. 